\title{
Uso del Teléfono Móvil, Juventud y Familia: Un Panorama de la Realidad Brasileña
}

\section{Using the Mobile Phone, Youth and Family: An Overview of the Brazilian Reality}

\author{
Fabiana Verza \\ Pontifícia Universidade Católica do Rio Grande do Sul, Brasil
}

\author{
Adriana Wagner \\ Universidad Federal do Rio Grande do Sul, Brasil
}

\begin{abstract}
Resumen. Este artículo se propone analizar el panorama del teléfono móvil en Brasil, desde una perspectiva psicosocial, considerando hacia el rol de los jóvenes y de la familia en el proceso de difusión de dicha tecnología en el país. Se presentan y discuten investigaciones sobre la juventud y el uso del teléfono móvil, describiendo los objetivos y motivaciones para su consumo, considerando que éste es el mayor sector consumidor de móviles en el país. Se describe el panorama de la juventud brasileña en el contexto de la Sociedad de la Información en Brasil. Se analiza la repercusión del uso de esta tecnología en las relaciones familiares, en las familias con hijos jóvenes, con el fin de comprender cómo padres e hijos manejan en sus relaciones el teléfono móvil en esta fase del ciclo vital.
\end{abstract}

Palabras clave: teléfono móvil, jóvenes, relaciones familiares, Sociedad de la Información.

\begin{abstract}
The main objective of this article is to analyze the use of mobile phones in Brazil, under a psychosocial perspective, focusing on the role of young people and the family in disseminating such technology in the country. It presents and discusses previous investigations on the use of mobile phones by young people, describing its aims and consumption motivations. Young people are the Brazil's main consumer group of mobile phone in the country. The article provides an outlook of Brazilian youth and its participation in the country's Information Society. The repercussions of the use of such technology in family relationships are analyzed, including families with young people. The objective is to understand how parent and teenagers handle mobile phone's technology in their relationships in this phase of the life cycle.

Key words: mobile phone, young, family relationship, Information Society.
\end{abstract}

\section{Introducción}

Aunque la aparición de la telefonía móvil haya sido en los años 70, solo fue en la década de los 80 cuando se inició su comercialización, ocurriendo su expansión en el mercado mundial en la década de los 90. Esas tres décadas estuvieron marcadas por la

La correspondencia sobre este artículo debe enviarse a la segunda autora a Rua Dr. João Dutra, 10/601 - Cep- 90630-100. Bairro Petrópolis. Porto Alegre - RS- Brasil. E-mail: adrianawagner.ufgrs @hotmail.com. evolución de la tecnología digital que llevó a la formación de la Sociedad de la Información. En ese contexto surge el paradigma de la tecnología de la información en el cual la cobertura, la complejidad y la disposición en red definen el modo en que se procesa la información en la actualidad. Como característica de este fenómeno, surge la convergencia de tecnologías para un sistema integrado (Castells, 2007). En ese proceso, el teléfono móvil puede considerarse el principal representante de la llamada convergencia tecnológica, ya que al integrar el texto, el audio, la imagen y los números en un 
mismo aparato, se inicia el consumo de informaciones multimedia y la diseminación de contenidos de estilo info-entretenimiento (Mantovani, 2006), y se intensifica el movimiento de digitalización de la vida cotidiana.

En Brasil, la venta de teléfonos móviles se inició en 1990, siete años después de su lanzamiento en el mercado mundial (Teleco, 2008). De acuerdo con la Agencia Nacional de Telecomunicación Anatel el rápido crecimiento del consumo de los móviles en el país, tuvo como factor determinante la privatización de la telefonía móvil, que hasta 1997 era un servicio estatal (Anatel, 2008). Según datos del último informe Teleco, Brasil es el quinto país en el ranking de consumo de teléfono móvil con un índice de 144,8 millones de aparatos distribuidos en una densidad de 76,22 móviles/100 habitantes (Teleco, 2008).

En una investigación sobre cómo la sociedad brasileña maneja las Nuevas Tecnologías de Información y Comunicación (NTICs), específicamente el teléfono móvil, Souza et al. (2006) identificaron que la población brasileña todavía utiliza la tecnología del teléfono móvil de manera sincrónica; es decir, los recursos del aparato son básicamente utilizados con el fin de establecer una comunicación interpersonal. Mientras tanto, los países europeos presentan un perfil de utilización asincrónica del móvil: acceso a Internet, descarga de imágenes, almacenamiento de archivos, entre otros, Souza, Jambeiro y Borges (2006).

En esta perspectiva, Castells (2007) distingue tres etapas evolutivas del uso de las nuevas tecnologías de telecomunicación. La primera se refiere a la automatización de las tareas, la segunda a la experiencia de su uso y la tercera a la reconfiguración de sus aplicaciones. Para llegar a la tercera etapa, los sujetos deben salir del rol de consumidores pasivos, volviéndose agentes productores de tecnología. Sin embargo, ese cambio depende de la etapa de desarrollo tecnológico de cada sociedad. En Brasil, Mantovani (2006) argumenta que las políticas de inclusión digital todavía no han avanzado más allá de la promoción del acceso a la infraestructura tecnológica. Predomina, de este modo, en el mercado consumidor brasileño de telefonía móvil, la búsqueda de la experimentación de esa tecnología sin todavía hacerse intentos de transformarla o de producirla.
Sin embargo, eso no significa que en Brasil el teléfono móvil no pueda ser considerado como uno de los principales medios de comunicación responsables de la difusión de la información y la expansión de la tecnología. Lo que interfiere, de hecho, en la forma sincrónica como se utiliza es básicamente el factor económico, pues el coste de esta tecnología en Brasil es más cara, comparativamente, con otros países (Rizzini et al., 2005). Brasil es considerado el primer país de América Latina en cuanto a mayor número de móviles (Teleco, 2008; Instituto Brasileño de Geografía y Estadística, IBGE, 2007), el número de domicilios con teléfono móvil $(59,3 \%)$ superó el de los que tienen teléfono fijo $(48,1 \%)$.

Desde esta perspectiva, Nicolaci-da-Costa (2004) apunta que el panorama brasileño de investigaciones sobre el tema del uso del teléfono móvil presenta algunas semejanzas con el panorama internacional, por dos motivos. El primero de ellos, la búsqueda de bases de datos nacionales revelan pocas investigaciones dirigidas al análisis de los impactos psicosociales del uso del móvil. En segundo lugar, se observa una constante recurrencia en la constatación de que los jóvenes representan siempre, en los diversos países donde ha habido este tipo de investigación, el mayor sector poblacional consumidor de esta tecnología.

Entre las investigaciones analizadas, un aspecto importante es la asociación entre el uso del teléfono móvil y la seguridad. Al igual que en otros países, las investigaciones en Brasil también indican que el teléfono móvil ofrece sensación de seguridad. Está comprobado que, desde la perspectiva de las relaciones familiares, el aparato puede servir como herramienta de auto-protección para los jóvenes y de monitorización por parte de los padres para con sus hijos (Nicolaci-da-Costa, 2004; Nicolaci-da-Costa 2007).

El factor de seguridad vinculado con el uso del teléfono móvil debe ser estudiado en el contexto brasileño, considerando las diferentes realidades socio-económicas y culturales que caracterizan el país. Aunque el acceso a la tecnología de manera general no sea facilitado en términos de igualdad para todos los jóvenes, es posible observar que, al menos en lo que concierne al teléfono móvil, éste es un objeto de consumo y de deseo que está presente 
en todos los estratos sociales. Una prueba de ello son los resultados de una investigación realizada con adolescentes de diversos países, en la cual se constató que los jóvenes brasileños son quienes más utilizan el teléfono móvil (81\%) en el mundo (Playground Digital, 2007).

En este sentido, puede pensarse que la tecnología forma parte de las necesidades de los jóvenes brasileños y las condiciones de acceso a los recursos tecnológicos se vuelven un aspecto de gran relevancia social en Brasil. La familia puede ser una alternativa para dar cuenta de esta demanda, principalmente en lo que se refiere al uso del teléfono móvil por parte de los jóvenes. Pero, ¿quiénes son los jóvenes de Brasil? ¿Qué representa el uso del móvil, por parte de los jóvenes, en la sociedad brasileña? El panorama del uso del teléfono móvil en Brasil puede trazarse tomando como punto de partida el análisis de quiénes son los protagonistas de la expansión de la utilización del teléfono móvil en el país: los jóvenes. A partir de ésto será posible comprender cuál es la repercusión de ese fenómeno en la esfera pública (sociedad) y privada (familia) y de qué manera contribuyen en el desarrollo de la Sociedad de la Información.

\section{Investigaciones en foco: juventud y teléfono móvil, la realidad brasileña}

En la actualidad ya es posible mapear en Brasil estudios psicosociales con poblaciones de diversos estados brasileños que tienen los más altos índices de consumo de aparatos móviles. Aunque los datos no representen la totalidad de la población brasileña, es posible conocer cómo la juventud de algunas regiones del país se maneja con esta tecnología.

En 2005 el canal MTV Brasil realizó el tercer Dossier Universo Joven con el objetivo de identificar tendencias, comprender y traducir las actitudes del joven brasileño, con edades entre 15 y 30 años. Fueron investigados 2359 jóvenes de todos los niveles culturales y estratos socio-económicos, residentes en las ciudades de Brasilia, Porto Alegre, São Paulo (capital e interior), Río de Janeiro, Belo Horizonte, Salvador y Recife (Dossiê Universo Jovem, 2005).
Los resultados de dicha investigación indicaron que la comunicación entre los jóvenes adquirió nuevos lenguajes y que hoy es posible seleccionar el tipo de canal de comunicación de acuerdo con lo que quiere decirse, a quién quiere decirse y en qué tiempo quiere decirse. Así, el teléfono móvil es considerado un utensilio básico para localizar a alguien y ser localizado $\mathrm{y}$, principalmente, representa una comunicación directa sin interlocutores que antecedan el contacto. En función de ello, la posesión del teléfono móvil aumentó vertiginosamente entre los años 1999 (Primer Dossier) y 2005. El uso de esta tecnología por parte del sector con mayor poder adquisitivo de la población joven brasileña, subió del 19\% en 1999 al $71 \%$ en 2005 y, aproximadamente el $79 \%$ de los jóvenes utiliza los mensajes de texto de su teléfono móvil para hablar con su grupo de amigos (Dossiê Universo Jovem, 2005).

En 2007, la red de televisión Nickelodeon Brasil realizó en doce países la investigación Playground Digital, con el objetivo de comprender de qué manera la tecnología influye en el estilo de vida de los niños, niñas y de los adolescentes, entre 8 y 14 años, así como la forma en que éstos reaccionan emocionalmente frente a los estímulos tecnológicos. Se observó que cada país utiliza la tecnología de modos diferentes. En Méjico, por ejemplo, el teléfono móvil es considerado un ítem de seguridad. Por otro lado, en Brasil, además de ser artículo útil para la seguridad, niños, niñas y adolescentes destacan como factor importante el estatus social que esta tecnología les otorga, considerando la posesión de un teléfono móvil un elemento de proyección social en la sociedad de consumo en donde está incluido (Playground Digital, 2007).

Es decir, al poseer un móvil, el joven se siente un participante activo en el medio en el que vive, se percibe seguidor de patrones establecidos por la cultura y, en consecuencia, siente pertenecer a ella. En esta fase del ciclo vital las tentativas de afirmar su identidad y de pertenencia a un grupo son prioritarios para los jóvenes. En este proceso, el teléfono móvil parece estar ayudando en esa tarea debido al valor social que se le otorga.

La industria brasileña de marketing se dio cuenta de esta dinámica en la sociedad de la información, e invierte en la población joven identificada como 
"nicho" potencial de inversión para nuevos productos. En un estudio realizado en 2008, denominado "Generación 90: Un nuevo conflicto entre generaciones" se constató que la juventud da demasiada importancia a los abordajes del marketing en el mercado de Brasil. El estudio investigó el comportamiento de jóvenes nacidos en los años 90, que hoy tienen entre 16 y 17 años, siendo éstos un total de 6,7 millones de habitantes, representando el 3,5\% de la población general. De éstos, el $22 \%$ trabaja, el $88 \%$ estudia y el $45 \%$ no vive con sus padres. Los resultados revelaron que la presencia de la tecnología en la vida diaria de los jóvenes se fortalece debido al creciente contacto con ésta y a la importancia que se atribuye a la vida virtual y a la realización de tareas múltiples - hacer varias cosas al mismo tiempo - en este rango de edades (O3 Digital, 2007).

Frente a esta circunstancia, puede percibirse que la practicidad del producto y la instantaneidad de la comunicación son aspectos potenciadores de la identificación del joven con esta tecnología, pues favorece elementos esenciales que el joven busca para sí en ese momento de su vida. La industria brasileña del marketing percibió esta relación e invierte en este sector al reconocer que el teléfono móvil impera entre sus actuales preferencias de consumo.

La investigación "Juventud Brasileña y Democracia: participación, esferas y políticas públicas”, investigó a 8.000 jóvenes de todos los niveles socioeconómicos y culturales, con edades entre 15 y 24 años, que viven en siete regiones metropolitanas y en el Distrito Federal, con el fin de conocer cuál es el perfil del joven, en lo que se refiere a la educación, situación familiar, trabajo, medios de comunicación y acceso a la cultura. El objetivo fue realizar una encuesta de opinión a través de Grupos de Diálogo, para que los jóvenes pudieran oírse mutuamente, expresar sus opciones e intercambiar experiencias sobre su situación en el país. En cuanto a su relación con los medios de comunicación, cultura, diversión e información, se observó que el 69,2\% de los jóvenes utiliza su tiempo libre yendo a los centros comerciales, el 85,8\% dijo estar informado a través de la televisión y el 51,2\% no tiene acceso al ordenador (IBASE, 2005). En esta investigación no fueron aportados datos sobre el uso del teléfono móvil. Sin embargo, considerando que más de $80 \%$ de la población joven tiene acceso a esta tecnología, ¿por qué todavía el móvil es un tema que se mantiene periférico en investigaciones que involucran medios de comunicación y juventud brasileña?

Según Mantovani (2006), la trayectoria evolutiva de los medios de comunicación siguió un movimiento dinámico iniciado en lo oral y hacia lo escrito, de lo escrito a la prensa, de la prensa a los medios electrónicos (radio y televisión) y de éstos evolucionó hacia las tecnologías digitales. Aunque en Brasil los medios electrónicos todavía predominen como uno de los recursos de entretenimiento más utilizados por la población, la tecnología digital, estando el teléfono móvil en cabeza, se expande aceleradamente, y el público joven brasileño puede ser considerado gran responsable de la expansión de la tecnología digital en el país.

No obstante, investigaciones nacionales dirigidas a la comprensión del uso del teléfono móvil en la adolescencia son todavía escasas, o en su gran mayoría, se limitan a sectores específicos de la población brasileña. Desde esta perspectiva, Rizzini et al. (2005) investigaron 949 jóvenes de la ciudad de Río de Janeiro, de estratos socio-económicos medios de la población, con edades entre 11 y 17 años, con el objetivo de conocer la interacción de los adolescentes con los nuevos medios de comunicación en sus espacios de convivencia y relaciones con la familia, la escuela y el grupo de amigos. Aunque los medios de comunicación más citados como preferidos por los adolescentes, hayan sido la televisión, el videojuego y juegos para ordenador, más de la mitad de los participantes de la investigación $(71,3 \%)$ manifestaron hacer uso del teléfono móvil y el $45,1 \%$ de estos declaró que utiliza este aparato durante la mayor parte del día.

También en Río de Janeiro fue desarrollada una investigación que pretendió evaluar cualitativamente el impacto psicológico del uso del móvil con 20 jóvenes de nivel socio-económico medio cuyas edades estaban entre 18 y 25 años. Los resultados de ese estudio señalan que el uso del teléfono móvil por parte de los jóvenes introduce nuevos indicadores de cambios micro-sociales que evolucionan hacia cambios psicológicos. Entre ellos predomina la necesidad de los jóvenes de tener el teléfono siempre cerca por el hecho de que el aparato les ofrece más liber- 
tad, autonomía, seguridad y privacidad. Otro resultado encontrado fue que el teléfono móvil permite acceso inmediato a la red social de apoyo de los jóvenes. Por esto, es considerado un recurso que facilita el nomadismo y cuando este falta produce una nueva forma de soledad: la soledad del sedentario que habita un mundo de nómadas (Nicolaci-daCosta, 2004).

Frente a estos datos la pregunta es: ¿Cuál es el espacio real que el teléfono móvil ocupa en la vida de los jóvenes brasileños? De acuerdo con Rizzini et al. (2005), el teléfono móvil todavía es utilizado por los jóvenes para relacionarse entre sí, con otros grupos y con la sociedad moderna. Sin embargo, la accesibilidad ofrecida por los recursos del teléfono móvil, los mensajes instantáneos, el acceso a Internet y a otros recursos de los medios de comunicación son grandes atractivos para quien quiere "buscar y ser buscado". Y, siendo la accesibilidad un aspecto importante de la vida social de los jóvenes, el impacto de tener un teléfono móvil en ese periodo de la vida es mayor que en el periodo de la vida adulta, pues ayuda a su proceso de socialización. Inclusive para los más tímidos, la posibilidad de llamar a los amigos sin tener que hablar con los padres de ellos, o enviar mensajes sin necesitar mirar directamente a los ojos, puede ser un recurso perfecto, facilitador de la interacción social (Muncer y Madell, 2005).

Considerando estos resultados, podemos observar que el teléfono móvil es muy valorado por los jóvenes brasileños debido a lo que representa. Esto es porque el hecho de disponer de un teléfono móvil demuestra un estilo de vida, un recurso de entretenimiento, una búsqueda del sentido de pertenencia y una forma que el joven encuentra para introducirse en el mundo. Ante la pluralidad de funciones de un móvil y la diversidad de jóvenes que lo utiliza, es posible considerar que la tecnología de la telefonía móvil se mueve aceleradamente acompañando el desarrollo de la Sociedad de la Información. Brasil actualmente ya forma parte de esta era de la movilidad, y el público joven es testigo activo de ese proceso. Por ello la importancia de conocer quiénes son los agentes de la expansión digital en el país.

La franja de la sociedad más representativa con respecto a la posesión de teléfono móvil es la pobla- ción joven que nació en los años 90, acompañada de la expansión del teléfono móvil en todo el mundo. Pero a fin de cuentas, ¿quiénes son los jóvenes brasileños de la actualidad? Y ¿por qué ellos son los mayores consumidores de teléfonos móviles en el país?

Los jóvenes brasileños representan un sector poblacional bastante significativo y tienen una importante participación en el proceso de desarrollo del país. Según la ONU, jóvenes son las personas que pertenecen al grupo de edad entre 15 y 24 años y Brasil asume ese patrón internacional de clasificación. De acuerdo con el último censo, la población joven comprende 35,2 millones y representa el $20 \%$ de la población brasileña (IBGE, 2007). Estos datos siguen la tendencia de América del Sur, que indican que ellos conforman el 20\%-25\% de la población suramericana (Informe Suramericano, 2008).

No obstante, en términos psicosociales, se dice que el país está en "condición juvenil". Se entiende por condición juvenil el modo como una sociedad se constituye y atribuye significado a ese momento del ciclo vital, considerándose el alcance social (clase, género, etnia), así como la dimensión históricogeneracional que circunda el ambiente donde el joven está siendo incluido (Abramo, 2005).

La condición juvenil brasileña es diversificada, pues varía de acuerdo con la situación socio-económica específica de cada grupo (Sarriera et al., 2007) y expresa las diferentes oportunidades disponibles para cada uno de estos grupos (IPEA, 2008). En ese sentido, la condición juvenil brasileña se caracteriza por la vida estudiantil (58\%) y por el mundo del trabajo, en el cual gran parte $(78 \%)$ tiene alguno o están en busca de uno (Venturi y Abramo, 2000).

También es posible observar que existe una relación entre el acceso a la tecnología del teléfono móvil y la situación socio-económica de la población brasileña de cada estado del país. En el año de 2005 el Instituto Brasileño de Geografía y Estadística (IBGE) realizó una Investigación Nacional mediante Muestra Domiciliar (PNAD) para conocer el acceso de la población brasileña a Internet y la propiedad de teléfonos móviles para uso personal. Los datos sobre la propiedad de teléfonos móviles, de acuerdo con los grupos de edad en el período de la juventud destacaron la región 
Centro-Oeste del Brasil con mayores índices de consumo (IBGE, 2007).

Dicha región está compuesta por los estados de Goiás, Mato Grosso, Mato Grosso do Sul y el Distrito Federal, en el cual se encuentra Brasilia, capital federal del país. La disponibilidad de teléfono móvil entre el grupo de edad de 11 a 14 años evidenció que en todos los estados brasileños, más del $50 \%$ de la población de estas edades dispone de esta tecnología, destacándose la región Centro-Oeste con un índice del $71,3 \%$ de propietarios de teléfonos móviles. En los grupos de edad entre 15 y 17 años, los índices se sitúan por encima del $80 \%$ en cada región de Brasil, destacándose nuevamente la región Centro-Oeste, con el 86,3\% de propietarios de estos aparatos (IBGE, 2007).

De acuerdo con el IBGE, el 65,2\% de los estudiantes de la población del Distrito Federal, tiene teléfono móvil. Éste es el índice más alto en el país. Frente a estos resultados, se constata que la facilidad de acceso al teléfono móvil en Brasil presenta altos índices de consumo en las regiones con los mejores indicadores sociales del país: Centro-Oeste: 47,5\%; región Sur (estados del Río Grande do Sul, Paraná y Santa Catarina): 47,6\% y región Sudeste (estados del Espírito Santo, Minas Gerais, Río de Janeiro y São Paulo): 41\% (IBGE, 2007).

Puede observarse que la adhesión de la población brasileña al teléfono móvil está relacionada a los índices de desarrollo socio-económico de cada región del país. Sin embargo, cuando se analiza de acuerdo con la edad, se observa que independientemente del estrato socio-económico al cual pertenece el joven, ese grupo considera que el teléfono móvil es un ítem de necesidad primaria. De este modo, la fuerte adhesión de la población joven a este bien de consumo debe ser analizada desde una perspectiva psicosocial, pues tal movimiento expresa cómo la cultura está respondiendo a este fenómeno. Según la ONU, los jóvenes pertenecen a una "cultura impulsada por los medios de comunicación" y se dice, incluso, que aquéllos que no tienen el deseo por tener los bienes y valores venerados por estos medios terminan perdiendo el derecho a soñar.

En ese sentido, lo que surgió inicialmente para ser simplemente una herramienta para facilitar y perfeccionar la comunicación entre las personas, terminó transformándose en un fenómeno que interfirió profundamente en las relaciones sociales en nuestro país. Esto ocurre porque la adquisición de un teléfono móvil entre la población joven puede representar el pasaporte hacia la libertad y la inserción grupal, o por el contrario, llevarlos a sentirse prisioneros y aislados. En la condición de la juventud, se busca pertenecer al grupo así como mejores formas de comunicación, pero ¿a qué precio? Frente a esta realidad, debe pensarse en qué condiciones el joven está adhiriendo masivamente al uso del teléfono móvil ¿Cuáles son las formas de acceso del joven a este recurso tecnológico? ¿Cuáles son sus prioridades? Y ¿cuál es el rol de la familia en este proceso?

\section{La repercusión del uso del teléfono móvil en las familias brasileñas: ¿cómo se da la relación padres-hijos-teléfono móvil?}

Los cambios introducidos por el teléfono móvil en las relaciones familiares fueron diversos. El teléfono móvil conquistó un espacio considerable en las familias con hijos adolescentes y acabó teniendo una participación activa en el proceso de independencia y construcción de la identidad de los jóvenes. La inserción de la tecnología en el día a día de las familias, instauró nuevos patrones de comunicación entre padres e hijos, volviendo más desafiadora la tarea de relacionarse en tiempos de las NTICs. Frente a esta realidad, ¿cómo manejan los padres y madres el teléfono móvil y las tareas del desarrollo típicas de las familias con hijos jóvenes?

Está comprobado que los padres no son los principales aliados para compartir con sus hijos asuntos relacionados a la tecnología en general (Casas et al., 2007) y lo mismo sucede cuando se habla del teléfono móvil (Malo, 2009). Una investigación que incluyó a Brasil y a otros cuatro países (España, India, África del Sur y Noruega) comparó la repercusión del teléfono móvil en las relaciones entre padres e hijos. Se entrevistaron 8.995 jóvenes con edades entre 12 y 16 años y 4.381 progenitores. Los resultados indicaron una tendencia de los progenitores a mostrarse menos interesados en relacionarse con los medios de comunicación que sus hijos, lo que, en consecuencia llevaba a que los jóvenes intercambia- 
ran informaciones sobre los medios con su grupo de iguales (Casas et al., 2003; Casas et al., 2007).

Sin embargo, la tecnología atraviesa las relaciones entre padres e hijos aunque la familia no sea el ambiente preferido por los jóvenes para tratar asuntos relacionados con ella. En cuanto al móvil y su utilización, debe intentarse comprender cuáles son las principales conexiones que relacionan padres e hijos en esa red de comunicación inalámbrica.

En el sistema familiar, la juventud de los hijos es una etapa que forma parte del ciclo vital evolutivo de la familia y demanda un aumento de la flexibilidad en la relación padres-hijos con el fin de fomentar la independencia de los jóvenes (Carter y McGoldrick, 2001). En este proceso, las fronteras familiares, que son barreras invisibles que regulan la permeabilidad para la entrada y salida de las emociones entre los miembros del sistema familiar (Ríos-González, 2003), deben también redefinirse. Las fronteras se destinan a la protección y diferenciación de los miembros en el contexto de la familia.

En esta dinámica, el teléfono móvil puede ser un gran aliado en la tarea de delimitar las fronteras entre padres y adolescentes. Esto sucede porque a partir de su inserción en las relaciones familiares se amplían las fronteras de comunicación del espacio real al espacio virtual (Nicolaci-da-Costa, 2004). En función de esto, las negociaciones entre padres e hijos no quedan restringidas solamente al espacio físico de la casa. Entonces, lo que ya era un desafío, se ha vuelto más complejo; es decir, ¿cómo establecer fronteras entre los miembros de la familia, frente a la tecnología?

El teléfono móvil permitió una mayor flexibilidad en los acuerdos y en el establecimiento de reglas, de tal modo que el hijo pudiese estar lejos y al mismo tiempo cerca de casa (Williams y Williams, 2005). Esta nueva tecnología representa para el joven un sendero en la comunicación, pues elimina la necesidad de intermediarios para hablar con quien desea; sobrepasa los límites de horario y lugar, el cual a veces no es favorecedor para las comunicaciones; así como burla las restricciones familiares para determinadas llamadas. Frente a esto, la actitud parental puede presentarse favorable o desfavorable con relación al uso del teléfono móvil por parte de los hijos. Su utilización es favorable cuando ayuda en las tareas educativas, en el proceso de separación entre padres e hijos y en el proceso de independencia de los jóvenes.

Con el fin de investigar la actitud de los progenitores frente al teléfono móvil y las nuevas culturas mediáticas emergentes entre sus hijos adolescentes, Malo (2009) realizó cinco grupos de discusión con un total de 41 participantes (padres, madres y grupos mixtos). Algunos progenitores destacaron los aspectos positivos de la tecnología argumentando que los medios audiovisuales pueden expandir las redes sociales de sus hijos, ofrecer nuevas posibilidades de trabajo y entretenimiento y facilitar el acceso a la información. Por otro lado, los progenitores también se mostraron contrarios a los cambios tecnológicos. Para muchos padres y madres sus hijos utilizan en exceso los medios audiovisuales, quedando repercutida la relación "cara a cara", así como sus habilidades lecto-escriptoras (Malo, 2009).

Un ejemplo lo observamos cuando el teléfono móvil favorece el ejercicio de la función parental, en donde los padres echan mano del móvil con el fin de controlar a sus hijos y ofrecer ayuda y protección "a larga distancia" (Ling y Haddon, 2001; Nicolaci-daCosta, 2006). Esta puede ser una alternativa saludable para que los hijos se sientan más seguros en el proceso de separación-individuación, teniendo en cuenta que este es un movimiento gradual y progresivo. Poseer un aparato de telefonía móvil, además de garantizar la sensación de seguridad y de protección, también puede ayudar en la tarea de "diferenciarse" de los padres y de "pertenecer" a un nuevo grupo (Katz y Sugiyama, 2007).

En muchas familias, por ejemplo, el joven adquiere un estatus diferente al regalársele un teléfono móvil. Simbólicamente, este aparato ayuda en el rito de paso hacia una nueva fase en el ciclo vital de la familia y del mismo joven. Esto ocurre porque regalarlo puede significar una actitud de confianza de los padres para con los hijos, que a su vez, a través de este ritual, se perciben listos para disfrutar de la autonomía y de la libertad que ellos desean (Lobet-Maris y Henin, 2002). Para muchas familias, el teléfono móvil puede simbolizar una extensión del cordón umbilical entre padres e hijos, una vez que la capacidad de conexión entre ellos es ampliada (Malo, 2009). Luego, un adolescente con teléfo- 
no móvil significa mayor tranquilidad para padres e hijos durante las salidas de casa, la experimentación de la vida nocturna, los viajes y otras tantas experiencias que se inauguran en esa fase de la vida del joven.

No obstante, la facilidad de los padres para acompañar la vida cotidiana de los hijos a través del uso del teléfono móvil también puede convertirse en un problema. El teléfono móvil, en sí mismo, no necesariamente determina patrones de comportamiento; pero su utilización puede interferir negativamente en las relaciones familiares. Es en ese momento que el uso del móvil puede ser considerado desfavorable en la relación padres-hijos. Algunos padres pueden "abusar" del control sobre los hijos a través del uso del teléfono móvil, mientras que los hijos pueden usarlo como una herramienta para liberarse del control parental (Ling, 2000). Considerando que la fase de la adolescencia puede favorecer la emergencia de una serie de conflictos dentro de la familia (Wagner, Falcke, Silveira y Mosmann, 2002), la forma de utilización del teléfono móvil puede ser un tema potenciador de situaciones estresantes entre todos los miembros del sistema familiar.

Conflictos típicos involucrando padres, hijos y teléfono móvil pueden suceder cuando la renta familiar no es compatible con el consumo del joven en llamadas, especialmente cuando éste no tiene recursos económicos propios para financiar sus gastos, por ejemplo. En esos casos, la utilización del sistema de tarjeta pre-pago de telefonía móvil es un recurso útil, ya que permite a los padres un control mayor sobre el gasto de los hijos y también permite a los hijos potenciar su auto-control y responsabilidad en los gastos generados por su móvil. En una investigación con 392 adolescentes, con edades entre 12 y 17 años, realizada en la ciudad de Viçosa (Minas Gerais) se encontraron cuatro grupos de jóvenes con diferentes características en relación al uso del teléfono móvil. En relación a la variable forma de pagar los gastos, predominó entre los iniciantes la opción por el sistema pre-pago en tres de los cuatro grupos. Solamente en el grupo con renta familiar más alta, se observó que eran los padres los responsables de pagar los gastos de los hijos en relación a su consumo (Luiz, 2008).

Se observa, por lo tanto, que el factor económico es importante en la decisión de regalar un teléfono móvil al hijo, especialmente, cuando no es éste quien paga la factura. Una alternativa para disminuir los gastos y mantener el contacto es la comunicación a través de mensajes de texto que además de ser más económicos, los intercambios de mensajes permiten una interacción no invasiva para con el hijo y posibilitan una comunicación en lugares donde no se escucha la voz a través del teléfono móvil, o en lugares donde no es posible hablar en voz alta. En ese sentido, la necesidad de estar disponible se reemplaza por medio de la tecnología del teléfono móvil y se vuelve positiva cuando las fronteras entre padres e hijos son respetadas.

Investigando sobre esa temática con 40 adolescentes y sus progenitores, con el fin de verificar los cambios en el ambiente familiar después de la introducción del teléfono móvil, Luiz (2008) observó que tanto los adolescentes como los padres tuvieron la misma percepción sobre los cambios en las relaciones familiares después de la inserción de esta tecnología en la vida del joven. Es decir, tanto padres como hijos mencionaron una mejoría en la comunicación y en las relaciones entre ellos $(27,5 \%$ desde la percepción de los hijos y $30 \%$ en la percepción de los padres) y resaltaron un aumento de conflictos con respecto al uso del aparato $(20 \%$ desde la perspectiva de los hijos y $6,67 \%$ desde la de los padres). Los hijos declararon sentir más libertad y confianza con relación a la familia $(15 \%)$ y los padres revelaron sentir más independencia con relación a los hijos $(6,67 \%)$. En relación a la sensación de seguridad y tranquilidad, el $50 \%$ de los padres destacó que este es el mayor factor de cambio ocurrido a partir de la introducción del teléfono móvil en las familias (Luiz, 2008).

Buscar la seguridad y tranquilidad con relación a los hijos, es constante entre los padres en esa fase del ciclo vital, y el teléfono móvil ha sido señalado como una tecnología capaz de proveer este aspecto. Una investigación realizada con 20 madres de adolescentes y adultos jóvenes con hijos con edades entre 18 y 25 años, de estrato socioeconómico medio de la ciudad de Río de Janeiro, estudió cómo ellas utilizaban el teléfono móvil con sus hijos. Se encontró una relación directa del uso con el control y la seguridad. Sin embargo, hubo diversas defini- 
ciones para esos conceptos por parte de las entrevistadas. Las madres argumentaron su uso con el fin de verificar la seguridad de sus hijos y controlar sus actividades por considerar que ése es su rol. Por otro lado, el intento de garantizar seguridad a los hijos se volvió ineficaz debido al contexto de violencia característico de los grandes centros urbanos. Frente a esa realidad, el teléfono móvil acabó ejerciendo la función principal de ayudar a combatir la angustia propia de las madres (Nicolaci-da-Costa, 2007).

Puede pensarse entonces, que el teléfono móvil es una tecnología de ayuda en la mediación del fenómeno relacionado con la seguridad de los hijos. Se observa también que el teléfono móvil ejerce un papel facilitador en la comunicación entre padres e hijos jóvenes; sin embargo, éste no modifica a las familias, sino que puede interferir positiva o negativamente en las relaciones entre sus miembros. Lo que ocurre con la interferencia de esa tecnología es una sofisticación de los elementos de entrada en el sistema familiar, pues en lo que respecta a sus tareas en función del desarrollo, la familia continúa siendo la misma. Desde esta perspectiva, el teléfono móvil en la familia debe encararse como un aparato que favorece la tarea básica de cuidar a los hijos y de promover su desarrollo evolutivo a lo largo del ciclo vital.

\section{Consideraciones finales}

A partir de las investigaciones sobre la población juvenil brasileña y a nivel internacional, puede constatarse que el teléfono móvil es una tecnología digital en constante expansión y su evolución en Brasil está vinculada a aspectos económicos y sociales. Los factores económicos interfieren no solamente en la forma como se utiliza el móvil, sino también en el desarrollo de investigaciones sobre este fenómeno. El coste de la tecnología y los diferentes niveles de poder adquisitivo de la población brasileña son dos factores importantes que deben ser tenidos en cuenta a la hora de comprender el panorama de la tecnología de la telefonía móvil en este país.

No obstante, ya es posible trazar un perfil descriptivo del usuario predominante del móvil en el país. Los jóvenes brasileños son el sector de la población que más tiene en su poder teléfonos móviles, y la adherencia a esa tecnología está asociada a la sensación de seguridad, especialmente cuando se analiza desde el interior de la esfera familiar. En este caso, el teléfono móvil es un gran aliado de la juventud brasileña y de sus familias.

En lo que se refiere a otros aspectos psicosociales, el teléfono móvil ha tenido un espacio en el proceso de construcción de la identidad del joven, en la medida en que se instala como un objeto de deseo y adquiere un valor subjetivo legitimado por la cultura del consumo. Su uso en la juventud está asociado, además de la búsqueda de la seguridad, también al ejercicio de la autonomía, la vivencia de la privacidad y a alternativas de entretenimiento. En este sentido el poseer un móvil aumenta la sensación de pertenencia al grupo y ofrece un estatus diferente frente a la sociedad, en función de su representación social. La literatura especializada indica que las representaciones sociales son constructoras y construidas por las practicas sociales; representan un objeto colectivamente compartido; son independientes de los objetos y de las personas que las representan y transforman los elementos del entorno en convenciones sociales que permiten la comunicación entre las personas (Casas, 2006). Luego, el móvil ocupa un espacio significativo en el imaginario juvenil.

En la llamada era de la movilidad donde se genera la sociedad de la información, se valora la comunicación virtual y la convergencia tecnológica, este aparato puede dar cuenta de diferentes funciones. El teléfono móvil, por ejemplo, reúne todas esas características a un coste y tamaño más asequible a la población, comparado con el ordenador. En realidad el teléfono móvil sigue una tendencia de evolución de los medios de comunicación en general, y deja de tener solamente su finalidad inicial de telecomunicación, ampliándose hacia un equipo de entretenimiento multifuncional. Tal vez esto pueda explicar por qué acabó volviéndose un utensilio indispensable para la gran mayoría de los jóvenes, a través del cuál se logran realizar desde tareas cotidianas hasta alcanzar un diferente estatus social frente a los distintos grupos que el joven pertenece, tales como la familia, los amigos, los compañeros de clase, entre otros. 
Las consecuencias de la innovación tecnológica originadas por el teléfono móvil, produjeron cambios significativos en el estilo de vida y en el comportamiento de las personas. En el caso de los jóvenes, debe reconocerse que su facilidad en manejar la tecnología puede ser utilizada como una manera de conquistar su propio espacio en el seno de la familia, así como un recurso que ayuda en su proceso de socialización y de inserción en la sociedad.

En este sentido, las familias deben estar atentas a los nuevos patrones de inserción social que pasaron a existir entre los jóvenes, en los tiempos de las Nuevas Tecnologías de la Información y Comunicación. Ese fenómeno generó una sofisticación en las relaciones padres-hijos en lo que se refiere a las tareas evolutivas del desarrollo de la adolescencia y todavía no hay respuestas para explicar esos cambios.

Desde la perspectiva del ámbito privado, es decir, de las relaciones familiares, el móvil, que debería entenderse como un instrumento de comunicación entre padres e hijos, terminó ocupando un espacio con dimensiones que van más allá de su función primaria que sería la de establecer una comunicación interpersonal. Entonces, puede pensarse que, si por un lado la expansión tecnológica en el interior de la esfera doméstica puede haber influenciado positivamente la adhesión de la sociedad al uso del móvil, del otro lado, se ampliaron las fronteras de comunicación hasta un punto del cual todavía no se sabe exactamente dónde está su límite.

El teléfono móvil pertenece a una tecnología que presupone la instantaneidad y virtualidad, es decir, se habla desde cualquier lugar con cualquier persona y en cualquier momento. Esa lógica alteró la noción de tiempo y de espacio en las relaciones interpersonales y como no parece ser un fenómeno pasajero, la importancia de aprender a manejar esa nueva dinámica se vuelve, así, fundamental.

Tal vez aquello que las familias y los jóvenes de la actualidad están revelando en tiempos de NTIC's, son las diversas posibilidades de integrar el desarrollo tecnológico y el desarrollo humano. Es decir, al ser utilizado como un recurso complementario al pasaje del ciclo vital de las familias con hijos jóvenes, la tecnología del aparato móvil también está en favor de la evolución de la sociedad y de las relaciones entre las personas.

Por lo tanto, la relación entre teléfono móvil y familias con hijos jóvenes es multifacética. Las finalidades referidas por padres e hijos a esta tecnología, son diferentes. Del mismo modo, también es diferente el impacto del uso del teléfono móvil en cada familia. Sin embargo, ya es posible percibir que el teléfono móvil interconecta las necesidades de padres e hijos y se conecta con las diversas realidades de las personas que viven en ese país.

En términos macro-sociales, es necesario desarrollar nuevos estudios sobre el uso del teléfono móvil, con el fin de comprender cómo este fenómeno está repercutiendo en la población brasileña, especialmente en lo que concierne a su relación con los jóvenes, con la familia y con la sociedad como un todo. A partir de estos estudios, será posible invertir en programas de intervención psicosocial que promuevan la concienciación de los efectos del progreso tecnológico en la sociedad, en la familia y en las nuevas formas de comunicación entre las personas.

El principal desafío, sin embargo, está en el hecho de que éste es un fenómeno que se procesa en la sociedad a ritmo acelerado y está en constante evolución. Siendo, tal vez en un futuro muy próximo, la Sociedad de la Información a la cual nos referimos hoy, ya esté tan integrada al modus vivendi, que imaginar las relaciones sin esta tecnología será como imaginar a la humanidad antes de la invención de la rueda. Cuando este tiempo llegue, el teléfono móvil seguramente será recordado como un aparato que agregó movilidad a la comunicación, traspasó las barreras del tiempo y el espacio, y añadió nuevas formas de ser y estar. 


\section{Extended Summary}

The last three decades have been marked by the evolution of digital technology all over the world, which led to the establishment of the Society of Information. In this context, the paradigm of the technology of information arises, in which the covering, the complexity and the network display define the way in which information is currently processed. As a characteristic of this phenomenon, the convergence of technologies for an integrated system appears (Castells, 2007). In this process, the mobile phone may be considered the main representative of the so called technological convergence, because, by integrating text, audio, image and numbers in the same device, it makes possible the use of multimedia information and the dissemination of info-entertainment contents (Mantovani, 2006), and it intensifies the digitalization of daily life.

This work aims at analyzing the panorama of the mobile phone in Brazil, from a psychosocial perspective, taking into consideration the role of youngsters and their families in the process of diffusion of this technology in the country. There has been a quick increase in the use of mobile phones in Brazil and, according to the last report by Teleco, Brazil is the fifth country in the ranking of mobile phone use, with 144,8 millions mobile phones distributed in a density of 76,22 phones/100 inhabitants (Teleco, 2008).

However, the policies of digital inclusion have not reached a point beyond the promotion of access to the technological infrastructure yet (Mantovani, 2006). It is predominant, thus, in the Brazilian mobile phone consumer market, the search for the experimentation of this technology, yet without aiming at transforming or producing it. It does not mean that the mobile phone in Brazil cannot be considered as one of the main means of communication responsible for the diffusion of information and for the expansion of technology. In fact, the economic factor interferes in the synchronic way in which it is used, since the costs of this technology is higher in Brazil than, comparatively, in other countries (Rizzini et al., 2005). Brazil is considered to be the country with the largest quantity of mobile phones in Latin America (Teleco, 2008; Brazilian Institute of Geography and Statistic, IBGE, 2007); the num- ber of homes with mobile phones $(59,3 \%)$ has overcome those which have fixed phones $(48,1 \%)$. From this perspective, Nicolaci-da-Costa (2004) claims that, in the Brazilian panorama, as well as internationally, young people represent the majority of consumers of this technology.

With the mobile phone, communication among young people has acquired new languages. It is possible to select the most appropriate channel of communication according to what one wishes to say, to whom one wishes to talk and when one wishes to communicate. Thus, the mobile phone is considered as a basic tool to be used in order to find someone or be found by someone; it primarily represents a direct communication, without the need of other speakers as mediators. Because of this, the use of mobile phones has increased vertiginously between 1995 and 2005. The use of this technology by Brazilian young people with higher purchasing power has developed from $19 \%$ in 1999 to $71 \%$ in 2005 , and approximately $79 \%$ of young people use text messages from their mobile phones in order to talk to their group of friends (Dossiê Universo Jovem, 2005).

That means that, by having a mobile phone, the youngster feels like he is an active participant of the environment in which he lives, he perceives himself as a follower of the established cultural patterns and, as a consequence, he feels that he is part of this culture. In this period of the life cycle, the attempts to affirm the identity and the participation in a group are priority for young people. In this process, the mobile phone seems to be helpful to this task, due to the high social value that it possesses.

The Brazilian marketing industry has noticed this dynamics in the information society and has focused its efforts on the young population, which is identified as a potential niche for new products. Considering these data, the question is: What is the real role that the mobile phone has in the life of young Brazilian people? According to Rizzini et al. (2005), the mobile phone is still used by youngsters in order to be in touch with one another, with other groups and with the modern society. Nevertheless, the accessibility offered by the resources of the mobile phone, the instant text messages, the access 
to internet and to other resources of the means of communication is enormously attractive to those who want to "search and be searched". And, being accessibility an important aspect of youngsters' social life, the impact of having a mobile phone in this period of life is greater than during adult life, since it helps their socialization process. Even for the timid ones, the possibility of calling their friends without having to talk to their parent's first or sending messages without having eye-contact may be a perfect resource which makes social interaction easier (Muncer and Madell, 2005).

It can be observed that the mobile phone is highly valued by young Brazilian people due to what it represents. This happens because the fact of having a mobile phone shows a lifestyle, an entertainment resource, a search for the sense of pertaining and a way found by the youngster to be introduced to the world. With the multiplicity of functions of a mobile phone and the diversity of youngsters that use it, it is possible to consider that the mobile phone technology evolves fast and accompanies the development of the Information Society. Brazil is currently part of this age of mobility, and the young people are active witnesses of this process. Considering this, it is important to know who the agents of digital expansion in this country are.

It can be observed that the adoption of the mobile phone by the Brazilian population is related to the socio-economic development rates of each region of the country. However, when the age factor is considered, it can be observed that, in spite of the socioeconomic stratum to which the youngster belongs, his group considers the mobile phone as an item of primary need. Thus, the strong adoption of this product by young people must be analyzed from a psycho-social perspective, since this movement expresses how culture is responding to this phenomenon. According to the UN, young people belong to a "culture propelled by the means of communication", and it is said, moreover, that those who do not have the wish of having the products and values venerated by the means of communication end up losing the right to dream.

In this sense, the device that was initially designed to be simply a tool that would facilitate and improve the communication among people ended up as a phenomenon which deeply interfered in the social relationships in our country. This happens because the purchase of a mobile phone by young people may represent a ticket to freedom and social insertion, or, on the contrary, may make them feel prisoners and isolated. During teenage years, one intends to belong to a group as well as to have better ways of communication, but to what cost? Considering this reality, one must think about the conditions in which the youngster is massively adopting the mobile phone. What are the forms of access of the young person to this technological resource? What are his priorities? And what is the role of the family in this process?

It is proved that the parents are not the main allies to share with children subjects related to technology in general (Casas et al., 2007a, 2007b) and the same happens when one speaks about the mobile phone (Malo, 2009). It is possible to identify a tendency of parents to show less interest in dealing with the means of communication than their children, which in consequence leads youngsters to exchange information on the means of communication within their peer group (Casas et al., 2003; Casas et al., 2007a, 2007b).

On the other hand, adolescence is part of the evolution of the family life cycle and demands an increase of flexibility in the relationship between parents and children in order to stimulate the independence of the youngsters (Carter and McGoldrick, 2001). In this process, the family borders, which are invisible barriers that regulate the permeability of emotions among the members of the family (RíosGonzález, 2003), must also be redefined. The borders are created so that the members of the family context can be protected and differentiated. In this sense, the mobile phone can be an important ally in the task of drawing the borders between parents and teenagers. This happens because, once the mobile phone is introduced in the family relations, the borders of communication go from the real space to the virtual space (Nicolaci-da-Costa, 2004). Because of that, the negotiations between parents and children are not uniquely restricted to the physical space of the house. Thus, what used to be a challenge has now turned into something much more complex; that is: how is it possible to establish borders among 
the family members, with the presence of technology?

The mobile phone has allowed a higher flexibility in agreements and in the establishment of rules in a way that the child can be away and, at the same time, near home (Williams and Williams, 2005). This new technology represents to the youngster a new way of communication, since it eliminates the need of having mediators in order to talk to other people; it transcends the limits of time and place, which sometimes are not favorable to communication; it as well breaks with family restrictions related to certain calls. Considering this panorama, the parental attitude may be presented as favorable or unfavorable regarding their children's use of mobile phone. The use of this device is favorable when it helps the teenager in educative tasks, in the process of separation between parents and children and in the process of independence of these youngsters.

One could conclude, thus, that the mobile phone is a helpful technology related to the security of children. Furthermore, it can be observed that the mobile phone adopts the role of facilitator of the communication between parents and teenagers; it does not modify the families, although it may interfere positively or negatively in the relationship of their members. What may occur with the introduction of this technology is a sophistication of the entrance elements in the familiar system, since the family, regarding its activities linked with development, is still the same. From this perspective, the mobile phone in the family must be seen as a device that favors the fundamental task of taking care of the children and promoting their evolutional development throughout the life cycle.

Conclusions:

The mobile phone is a technology in constant expansion and its evolution in Brazil is related to economic and social aspects;

Young Brazilian people are the part of the population which has the highest number of mobile phones, and the adoption of this technology is related to a sense of security, especially within the family group. In this case, the mobile phone is a great ally of the Brazilian youngsters and their families;

Its use by teenagers is associated, besides in order to have a sense of security, to the exercise of auton- omy, to privacy and to alternative ways of entertainment. In this sense, having a mobile phone increases the sensation of belonging to a group and permits the adoption of a different status within the society, because of its social representation;

The relation between the mobile phone and families with children is multifaceted. However, it is possible to perceive that the mobile phone interconnects the necessities of parents and children and is related to the diverse realities of the people who live in this country.

\section{Referencias}

Abramo, H. (2005). Condição juvenil no Brasil contemporâneo. En H. Abramo, P.P.M. Branco (Eds.). Retratos da juventude brasileira: análise de uma pesquisa nacional (pp. 37-73). San Paulo: Instituto Cidadania; Fundação Perseu Abramo Anatel (2008). Indicadores. Agência Nacional de Telecomunicação. Obtenido el 23 de Mayo de 2008 de http://www.anatel.gov.br/indicadores.

Carter, B. y Mc Goldrick, M. (2001). As mudanças no ciclo de vida familiar. Porto Alegre: Artes Médicas.

Casas, F. (2006). Infancia y representaciones sociales. Política y Sociedad, 43, 27-42.

Casas, F.; Figuer, C.; González, M. y Malo, S. (2007a) Los medios audiovisuales entre los progenitores y los hijos e hijas. Cultura y Educación, 19, 311-330.

Casas, F.; Rizzini, I.; September, R.; Mjaavatn, P. E. y Nayar, U. (2007b). Adolescents and Áudio-visual Media in Five Countries. Documenta Universitaria. Girona: UDG Publicacions.

Casas, F.; Gonzalez, M. y Figuer, C. (2003). Parents, Children and Media: Some Data from Spain, Brazil, Norway, South Africa and India. En C. von Feilitzen y U. Carlsson (Eds). Promote or Protect? Perspectives on Media Regulations. Götemburg: The International Clearinghouse on Children, Youth and Media - NORDICOM Göteborg University.

Castells, M. (2007). A Sociedade em rede: a era da Informação, Economia, Sociedade e Cultura. Volume I. San Paulo: Paz e Terra. 
Dossiê Universo Jovem (2005). Projeto MTV Brasil. Obtenido el Marzo de 2008 de http://portal. ibta.com.br/cursos/ibtanews/ibtanews_5.

IBASE (2005). Juventude Brasileira e democracia participação, esferas e políticas públicas. Relatório final. Rio de Janeiro: Instituto Brasileiro de Análises Sociais e Econômicas. Obtenido el 25 de Julio de 2008 de http:// www.ibase.br/pubibase/media/ibase_relatorio_ju ventude.pdf.

IBGE (2007). Pesquisa nacional por amostra de domicílios 2005 - Acesso a internet e posse de telefone móvel para uso pessoal. Rio de Janeiro: Instituto Brasileiro de Geografía e Estatística. Obtenido el 15 de Juño de 2008 de http:// www.ibge.gov.br/home/estatistica/populacao/ace ssoainternet/internet.pdf.

Informe Sudamericano (2008). Juventud e integración sudamericana: caracterización de situaciones tipo y organizaciones juveniles. Demandas para construir una agenda común. Obtenido el 20 de septiembre de 2008 de http//www.ibase.br/userimages/RelatorioJuventude08_Sul_americano.pdf

IPEA (2008). Política Social e Desenvolvimento - A Juventude em Foco. Políticas Sociais - acompanhamentos e análise. Obtenido el 18 de Agosto de 2008.

http://www.ipea.gov.br/sites/000/2/publicacoes/bps ociais/bps_15/02_politicasocialdesenvolvimento. pdf.

Katz, J. y Sugiyama, S. (2006). Mobile phones as fashion statements: evidence from student surveys in the US and Japan. New Media \& Society, 8, 321-337.

Lobet Maris, C. I y Henin, J. (2002). Hablar sin comunicar o comunicar sin hablar: del GSM al SMS. Juventud y teléfonos móviles, Revista de estudios de juventud, 57, 101-114.

Ling, R. (2000). We will be reached: the use of mobile telephony among Norwegian youth. Information Technology and People, 13, 102120.

Ling, R. y Haddon, L. (2001). Mobile telephony, mobility and the coordination of everyday life. Obtenido el 25 de Abril de 2007 de

http://www.telenor.no/fou/program/nomadiske/articles/rich/(2001)Mobile.pdf
Luiz, G. V. (2008). Consumo de telefone celular: significados e influências na vida cotidiana dos adolescentes. Dissertação (Mestrado em Economia Doméstica) - Programa de PósGraduação em Economia Familiar, Universidade Federal de Viçosa, Minas Gerais.

Malo, S. (2009). Culturas mediáticas adolescentes: Un estudio psicosocial centrado en el teléfono móvil. Servidor de tesis doctorales en red (TDX). http://www.tdx.cat/TDX-0223109-134709. Depósito Legal: GI-302-2009

Mantovani. C. M. C. A. (2006). Info-entretenimento na telefonia celular: informação, mobilidade e interação social em um novo espaço de fluxos. Dissertação (Mestrado em Ciência da Informação) - Escola de Ciência da Informação, Universidade Federal de Minas Gerais, Belo Horizonte, Minas Gerais.

Muncer, S. y Madell, D. (2005) Are Internet and Mobile Phone Communication Complementary Activivities amongst Young People? A study from a "rational actor" perspective. Information, Communication \& Society, 8, 64-80.

Nicolaci-da-Costa, A. M. (2004). Impactos Psicológicos do Uso de Celulares: Uma pesquisa Exploratória com Jovens Brasileiros. Psicologia: Teoria e Pesquisa, 20 (2) 165-174.

Nicolaci-da-Costa, A. M. (2007). Celulares: um "presente do céu" para as mães de jovens. Psicologia e Sociedade, 19, 108-116.

Nicolaci-da-Costa, A. M. (2006). Celulares: a emergência de um novo tipo de controle materno. Psicologia e Sociedade, 18, 88-96.

O3 Digital (2007). Geração "90” investiga relação entre jovens e mídias. Obtenido el 15 de Septiembre de 2007 de http//www.o3.com.br/ noticias.

Playground Digital (2007). A relação das crianças com a tecnologia. Obtenido el 7 Julio de 2008 de http//www.maxpessnet.com.br/e/estrategia.

Ríos Gonzáles, J. A. (2003) Vocabulario Básico de Orientación y Terapia Familiar. Madrid: Editora CCS.

Rizzini, I. Pereira, L., Zamora, M. H., Coelho, A. F., Winograd B. y Carvalho, M. (2005). Adolescentes brasileiros, mídia e novas tecnologias. ALCEU, 6, 41-63. 
Sarriera, J. C., Tatim, D., Coelho, R. P .S. y Bücker, J. (2007). Uso do tempo livre por adolescentes de classe popular. Psicologia: Reflexão e Crítica, 20, 361-367.

Souza, L. S.; Jambeiro, O. y Borges, J. (2006). Cidades Digitais, Telefonia Móvel e Interação Social na Sociedade Brasileira Contemporânea. Obtenido el 10 de Mayo de 2007de http://www. razonypalabra.org.mx/anteriores/n49/mesa2.html).

Teleco (2008). Quadro: Em Debate. Telecomunicações Brasil. Obtenido el 16 de Mayo de 2008 de http://www.teleco.com.br/emde bate/qudros 02.asp.
Teleco (2008) Relatórios. Telecomunicações Brasil. Obtenido el 16 de Mayo de 2008 de http://www.teleco.com.br/relatorios.asp.

Venturi, G. y Abramo, H. (2000) Juventude, política e cultura. Teoria \& Debate, 45, 28-33.

Wagner, A.; Falcke, D.; Silveira, L. M. B. Y Mosmann, C. P. (2002). A Comunicação em Famílias com Filhos Adolescentes. Psicologia em Estudo, 7 (1), 75-80.

Williams, S. y Williams, L. (2005). 'Space Invaders: The negotiation of teenage boundaries through the mobile phone'. Sociological Review, 53 (2), 314-333.

Manuscrito recibido: $11 / 01 / 2010$

Revisión recibida: 23/01/2010

Manuscrito aceptado: 26/01/2010 